\title{
A 17-year-old male with an unusual case of peritonitis
}

$\mathrm{A}_{\mathrm{t}}^{1}$

17-year-old male presented to the emergency department with a 24-hour history of increasing epigastric pain, black stools and bilious vomiting. He had a 5-month history of vague epigastric discomfort and a 1-2month history of anorexia, weight loss $(6.8 \mathrm{~kg})$, night sweats, occasional dizziness and shortness of breath. He had immigrated to Canada from the Philippines 3 years previously.

On physical examination the patient was found to be hypotensive (blood pressure 87/57 $\mathrm{mm} \mathrm{Hg}$ ), tachycardic (heart rate 163 beats/min) and slightly tachypneic, with normal oxygen saturation. He was afebrile but was pale, cachectic and appeared generally unwell. He had diffuse abdominal rebound tenderness and guarding and no audible bowel sounds. A digital rectal examination revealed melena stool that was positive for occult blood. A nasogastric tube was placed and drained bilious fluid. The rest of his examination was unremarkable. Baseline laboratory tests showed anemia (hemoglobin level $96 \mathrm{~g} / \mathrm{L}$ ) with leukocytosis (cell count $25.9 \times$ $10 \% / \mathrm{L})$ and mild thrombocytosis (cell count $675 \times 10^{9} / \mathrm{L}$ ).

At this point, the differential diagnosis included appendicitis, inflammatory bowel disease, and malignant disease such as lymphoma; however, a fungal or tubercular infection could not be ruled out. A CT scan of the abdomen with oral and intravenous contrast media revealed a mass-like lesion in a dilated small-bowel loop (Fig. 1, arrow). Exploratory laparotomy revealed a necrotizing mass and no evidence of perforation, in-

$\infty$ flammatory bowel disease or appendicitis. Biopsy of the specimen confirmed necrotizing granulomatous inflammatory tissue with acid-fast bacili identified as Mycobacterium tuberculosis. The patient's gastrointestinal symptoms resolved a few weeks

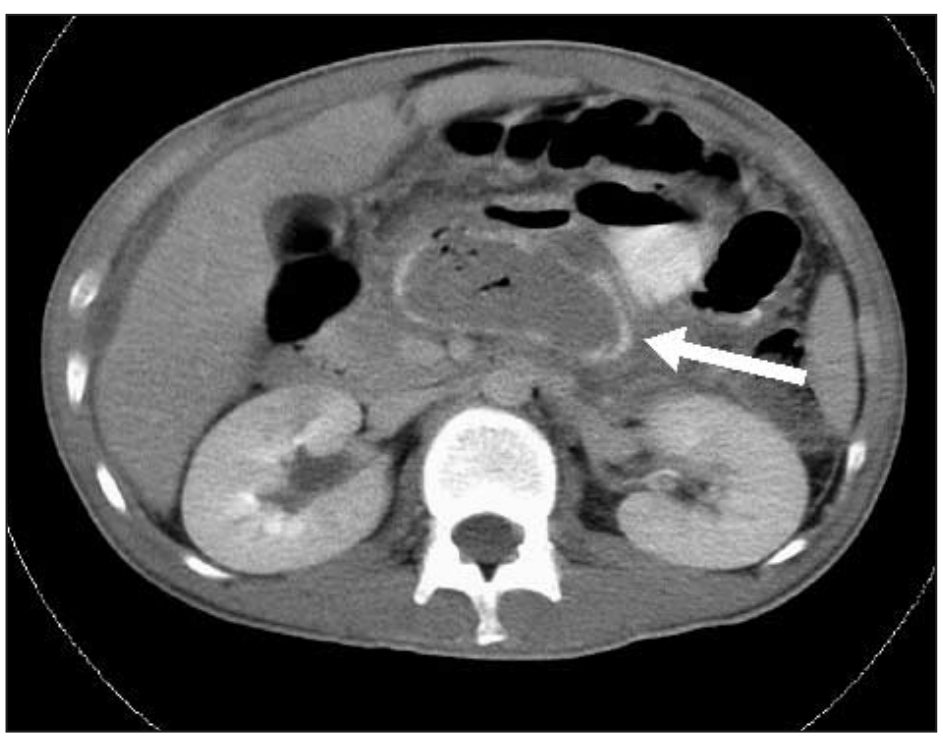

after initiation of treatment with rifampin, isoniazid, pyrazinamide and ethambutol.

About 1 billion people have tuberculosis (TB) worldwide and 8.7 million new cases develop each year, according to estimates of the World Health Organization. Of the new cases, $15 \%$ involve children and result in 450000 childhood deaths annually. ${ }^{1}$ In 2001 , there were 1704 cases (5.5 per 100000$)$ of new and relapsed cases of TB reported in Canada; ${ }^{2} 6.5 \%$ involved individuals less than 15 years old ${ }^{2}$ and $62.3 \%$ involved immigrants. Extrapulmonary TB accounts for about $11 \%$ of cases of new TB in Canada and may involve lymphatic, genitourinary, gastrointestinal, cardiovascular, skeletal or central nervous systems. ${ }^{3}$ Immigrants from TB-endemic countries and HIV-positive patients are at greatest risk of gastrointestinal $\mathrm{TB},{ }^{4}$ but our patient, with his consent, tested negative for HIV infection.

Presenting symptoms of gastrointestinal TB may include abdominal distension, low-grade fever, anemia and weight loss, but presentation is often insidous and may be nonspecific. In children, ascites and visible peristalsis have also been reported. ${ }^{5}$ Gastrointestinal TB may occur anywhere in the gut, but the ileocecum, ileum and colon are the most common sites. Laparotomy is usually needed for diagnosis. Treatment requires at least 9 months of standard drug therapy. ${ }^{3}$ Although abdominal $\mathrm{TB}$ is rare, it may lead to peritonitis and death if left undiagnosed. A high index of suspicion should be retained in the evaluation of nonspecific complaints in susceptible populations.

\section{Ronik Kanani}

Aleixo Muise

Department of Paediatrics

Hospital for Sick Children

University of Toronto

Toronto, Ont.

\section{References}

1. World Health Organization. Communicable disease cluster. Global tuberculosis report for 2002. Geneva: The Organization; 2002.

2. Tuberculosis in Canada 2001. Ottawa: Health Canada; 2001. Cat no H49/pphb-dgspsp/publicat/tbcan01/ (accessed 2004 Apr 6).

3. Fanning A. Tuberculosis: 6. Extrapulmonary disease. CMAf 1999;160(11): 1597-603.

4. Marshall JB. Tuberculosis of the gastrointestinal tract and peritoneum. Am 7 Gastroenterol 1993;88(7):989-99.

5. Talwar BS, Talwar R, Chowdhary B, Prasad P. Abdominal tuberculosis in children: an Indian experience. 7 Trop Pediatr 2000;46(6):368-70. 108/2001. Available: www.hc-sc.gc.ca 